\title{
Efeitos da supressão da aspiração endotraqueal na incidência de complicações pulmonares no pós operatório de cirurgia cardíaca
}

\section{Effects of suppression of endotracheal suctioning in the incidence of pulmonary complications on the postoperative cardiac surgery}

\author{
Andressa Campos ${ }^{1}$ (i) \\ Amanda Braga de Louredo Rabelo ${ }^{2}$ \\ Juliana Oliveira Barros ${ }^{3}$ \\ Emilia Nozawa ${ }^{4}$ (1) \\ Ludhmila Abraão Hajjar 5 (1) \\ Filomena Regina Barbosa Gomes Gallas 6 (1) \\ Maria Ignêz Zanetti Feltrim ${ }^{7}$ (1)
}

\begin{abstract}
1,3-7Instituto do Coração, Hospital das Clínicas, Universidade de São Paulo (São Paulo). São Paulo, Brasil. hdressa@ig.com.br, juliannaoliveir@hotmail.com, emilia.nozawa@incor.usp.br, ludhmila@usp.br, filomena.galas@hc.fm.usp.br, mi.feltrim@incor.usp.br ${ }^{2}$ Autora para correspondência. Instituto do Coração, Hospital das Clínicas, Universidade de São Paulo (São Paulo). São Paulo, Brasil. amanda_louredo@hotmail.com
\end{abstract}

RESUMO | INTRODUÇão: Aspiração traqueal é utilizada rotineiramente após cirurgia cardíaca para garantir adequada ventilação. OBJETIVO: Verificar se a ausência da aspiração traqueal antes da extubação em pacientes sem sinais de secreção brônquica influencia na incidência de complicações pulmonares, além de repercussões hemodinâmicas e ventilatórias do procedimento e custos. MÉTODOS: Pacientes foram avaliados entre agosto/2012 e julho/2014, divididos igualmente em Grupo ASP (aspiração traqueal prévia à extubação) e NASP (sem aspiração prévia). Foram incluídos indivíduos com: primeira cirurgia cardíaca, idade entre 18 e 75 anos, IMC $\leq 30 \mathrm{~kg} / \mathrm{m}^{2}$ e sem doença pulmonar prévia. Foram excluídos indivíduos com tempo de CEC > 120 minutos, necessidade de assistência circulatória mecânica, relação $\mathrm{PaO} / \mathrm{FiO} 2<$ $200, \mathrm{SpO} 2<92 \%$, tempo de intubação $>12$ horas, PAM $<60 \mathrm{mmHg}$ e sinais de secreção pulmonar. Variáveis hemodinâmicas, ventilatórias, complicações pulmonares e custos hospitalares foram avaliados. RESULTADOS: Foram analisados 228 pacientes (ASP= 114 NASP=114). FC, PAS, PAD e PAM elevaram-se após aspiração e pós extubação nos grupos, retornando aos valores basais no decorrer do tempo. Não houve diferença estatística na FR e SpO2. Complicações pulmonares representaram 7,8\%, sem diferença entre os grupos. CONCLUSÃO: A supressão da aspiração traqueal nos pacientes em pós-operatório imediato de cirurgia cardíaca não influenciou na incidência de complicações pulmonares e evolução clínica pós-operatória. Durante aspiração, houve alterações hemodinâmicas e respiratórias, sem repercussões clínicas, porém estas mesmas alterações ocorreram no grupo NASP após extubação, em menor intensidade. Aspiração endotraqueal sem indicação mostrou-se custosa e com desperdício de tempo dos profissionais.

PALAVRAS-CHAVE: Aspiração traqueal. Cirurgia cardiovascular. Complicações pulmonares. Fisioterapia.

\begin{abstract}
INTRODUCTION: Endotracheal suction is routinely used after cardiac surgery to ensure adequate ventilation. OBJECTIVE: To verify if that the absence of endotracheal suctioning before extubation in patients without signs of bronchial secretion have influence on the incidence of pulmonary complications, beyond hemodynamic and ventilatory repercussions of the procedure and costs. METHODS: Patients were evaluated between August/2012 and July/2014, divided equally into ASP Group (tracheal aspiration prior to extubation) and NASP (without prior aspiration). Individuals with: first cardiac surgery, age between 18 and 75 years, $\mathrm{BMI} \leq 30 \mathrm{~kg} / \mathrm{m}^{2}$ and without previous lung disease were included. Individuals with ECC time $>120$ minutes, need for mechanical circulatory assistance, $\mathrm{PaO} 2$ / FiO2 ratio $<200$, SpO2 $<92 \%$, intubation time $>12$ hours, MBP $<60 \mathrm{mmHg}$ and signs of pulmonary secretion were excluded. Hemodynamic, ventilatory variables, pulmonary complications and hospital costs were assessed. RESULTS: 228 patients were analyzed $(\mathrm{ASP}=114 \mathrm{NASP}=114) . \mathrm{HR}$, SBP, DBP and MBP increased after aspiration and post extubation in the groups, returning to baseline values over time. There was no statistical difference in RR and SpO2. Pulmonary complications accounted for $7.8 \%$, with no difference between groups. CONCLUSION: The suppression of tracheal suctioning in patients in the immediate postoperative period of cardiac surgery did not influence the incidence of pulmonary complications and postoperative clinical evolution. During aspiration, there were hemodynamic and respiratory changes, with no clinical repercussions, but these same changes occurred in the NASP group after extubation, to a lesser extent. Endotracheal suctioning without indication proved to be costly and wasted professionals' time.
\end{abstract}

KEYWORDS: Suction. Pulmonary atelectasis. Thoracic surgery. Health care costs. Physiotherapy. 


\section{Introdução}

A intubação endotraqueal, comumente utilizada em cirurgia cardíaca, assegura ao paciente abertura e manutenção das vias aéreas, garantindo adequada ventilação e monitorização, porém impossibilita a mobilização e expectoração das secreções brônquicas de forma efetiva ${ }^{1}$. A aspiração de secreções pulmonares no paciente com via aérea artificial é realizada para garantir sua perviabilidade ${ }^{2}$, prevenindo obstruções no trato respiratório, que provocam, principalmente, aumento do trabalho ventilatório, atelectasia e infecções pulmonares ${ }^{3,4}$.

É um procedimento de rotina e de alta aplicabilidade, porém pode causar alguns efeitos indesejáveis, como a diminuição da complacência dinâmica e da capacidade residual funcional, atelectasia, hipoxemia, trauma de mucosa brônquica, tosse excessiva, broncoespasmo, instabilidade cardiovascular, aumento da pressão intracraniana, dor, ansiedade, dispneia e arritmias cardíacas ${ }^{5-7}$.

Essas complicações podem estar acentuadas em indivíduos pós-cirurgia cardíaca, devido às alterações hemodinâmicas causadas pelo ato cirúrgico, doença cardíaca prévia, tempo de cirurgia, tipo de procedimento realizado, que contribuem para o aparecimento de arritmias cardíacas, sangramentos, hipoxemia e diminuição do débito cardíaco.

Desta forma, a realização da aspiração traqueal deve ser indicada somente quando necessária. A presença de secreção brônquica pode ser detectada pela ausculta de sons pulmonares como roncos e estertores, presença de secreção no tubo orotraqueal ou presença de padrão denteado na curva fluxo/volume e aumento do pico de pressão inspiratória, ambos visualizados no monitor do ventilador mecânico .

Em nosso meio, rotineiramente, indivíduos no pósoperatório imediato são submetidos à aspiração traqueal e nasotraqueal, previamente à extubação. No entanto, parte desses sujeitos evolui com breve tempo de ventilação mecânica e não apresentam sinais indicativos de retenção de secreção brônquica.

Este estudo se propôs a avaliar a hipótese de que a supressão da aspiração traqueal pré-extubação em indivíduos sem sinais de secreção brônquica, no pós-operatório imediato de cirurgia cardíaca, não influencia a incidência de complicações pulmonares.
Para complementar, estudamos as repercussões hemodinâmicas e ventilatórias provocadas pelo procedimento envolvendo a extubação, além dos custos do procedimento.

\section{Métodos}

\section{Casuística}

Trata-se de um estudo prospectivo, observacional e randomizado, realizado entre agosto de 2012 e julho de 2014, nas UTI cirúrgicas do Instituto do Coração do Hospital das Clínicas da Universidade de São Paulo (CAAE 11855413.3.0000.0068). Participaram 228 indivíduos, randomizados igualmente em: Grupo ASP (extubação com execução prévia da aspiração traqueal e nasotraqueal) e NASP (extubação sem execução prévia da aspiração traqueal e nasotraqueal).

Foram incluídos aqueles de primeira cirurgia cardíaca, com idade entre 18 e 75 anos, índice de massa corpórea $(I M C) \leq 30 \mathrm{~kg} / \mathrm{m}^{2}$ e ausência de doença pulmonar prévia. Foram excluídos os sujeitos que apresentaram tempo de circulação extra corpórea (CEC) maior que 120 minutos, necessidade de assistência circulatória mecânica, relação $\mathrm{PaO2} / \mathrm{FiO} 2$ menor que 200, saturação periférica de oxigênio (SpO2) menor que $92 \%$, tempo de intubação maior que 12 horas, pressão arterial média (PAM) menor que $60 \mathrm{mmHg}$, e aqueles com secreção brônquica caracterizado pela presença de padrão denteado na curva de fluxo, secreção na cânula endotraqueal ou ausculta pulmonar com ruídos adventícios do tipo roncos.

\section{Protocolo}

Os indivíduos em lista cirúrgica foram convidados a participar do estudo e após serem informados do protocolo, os concordantes assinaram o Termo de Consentimento Livre e Esclarecido.

No momento da admissão na Unidade de Terapia Intensiva (UTI) foram inseridos aqueles que preenchiam os critérios do estudo. Os indivíduos estavam intubados e ventilados por meio do ventilador mecânico (Galileo $\circledR_{\text {, Raphael }}$ ou Avea $^{\circledR}$ ), com parâmetros ajustados para garantir SpO2 maior ou igual a 95\%.

Na ausência de alterações respiratórias, metabólicas, hemodinâmicas ou neurológicas, iniciou-se o 
programa de retirada gradual do suporte ventilatório. No momento pré-extubação os indivíduos foram randomizados em dois grupos:

- GRUPO ASP: com o paciente em posição sentada ou semi sentada, procedeu-se ao aumento da fração inspirada de oxigênio para 1,0 por um minuto e após, verificado a SpO2. Executou-se a técnica de aspiração traqueal e em seguida, o paciente foi reconectado ao ventilador, aguardando-se o retorno da SpO2. Realizou-se aspiração nasotraqueal, seguida de aspiração da cavidade oral. Ao final, com o paciente realizando inspiração máxima, o cuff foi desinsuflado, retirando-se o tubo endotraqueal. Após, instalou-se cateter nasal de oxigênio, fluxo de até $5 \mathrm{~L} / \mathrm{min}$, para manter $\mathrm{SpO} 2$ maior ou igual a $95 \%$.

- GRUPO NASP: com o paciente em posição sentada ou semi sentada, desconectou-se o ventilador mecânico; o cuff foi desinsuflado e solicitou-se inspiração máxima, com retirada do tubo endotraqueal. A seguir solicitou-se tosse do paciente. Instalado cateter nasal de oxigênio, fluxo de até $5 \mathrm{~L} / \mathrm{min}$ para manter SpO2 maior ou igual a 95\%.

Em ambos os grupos calculou-se o tempo despendido no procedimento, iniciado no momento da desconexão do paciente com o ventilador mecânico até o término da instalação da oxigenoterapia.

As variáveis hemodinâmicas de pressão arterial sistólica (PAS), pressão arterial diastólica (PAD), pressão arterial média (PAM), frequência cardíaca ( $F C$ ), foram observadas diretamente nos monitores a beira leito (Dixtal ${ }^{\circledR}$ e Philips ${ }^{\circledR}$ ). A medida da frequência respiratória (FR) foi obtida por meio da observação do movimento torácico. Essas variáveis e a SpO2, foram coletadas nos momentos pré-extubação, imediatamente à extubação e de minuto a minuto, nos 10 primeiros minutos imediatamente após o procedimento e 30 e 60 minutos após.

Todos os sujeitos foram acompanhados durante seu período hospitalar, para registro das complicações pós-operatórias, classificadas em infecciosas, hemodinâmicas, neurológicas e outras. As infecciosas pulmonares foram classificadas em traqueobronquite e pneumonia, segundo critérios da Comissão de Controle de Infecção Hospitalar̊ sendo:

- Traqueobronquite: definida pela presença de febre (temperatura maior ou igual a $37,5^{\circ} \mathrm{C}$ ), aumento da quantidade de secreção pulmonar com seu aspecto se tornando purulento e sem novo infiltrado no parênquima pulmonar, avaliado por meio da radiografia de tórax;

- Pneumonia: confirmada por pelo menos dois dos critérios: alteração clínica com estertores crepitantes pela ausculta pulmonar ou macicez à percussão, somado a um dos seguintes achados: 1) microrganismo isolado em hemocultura; escarro purulento ou mudança nas características do escarro; isolamento de microrganismo na amostra obtida por biópsia ou lavado bronco alveolar: 2) alteração do exame radiológico de tórax, evidenciando infiltrado novo ou progressivo, consolidação, cavitação ou derrame pleural.

A análise do custo do procedimento baseou-se nos materiais utilizados no procedimento de aspiração e no tempo despendido pelos profissionais envolvidos. No Grupo ASP foram utilizados seringa de $20 \mathrm{ml}$, sonda de aspiração com válvula número 12 , coletor de secreção de vias aéreas, luva estéril, luva de procedimento e máscara cirúrgica. Os materiais utilizados no Grupo NASP foram: luva de procedimento, máscara cirúrgica e seringa de $20 \mathrm{ml}$. A aspiração traqueal foi sempre realizada por dois profissionais.

\section{Análise Estatística}

A equivalência de ocorrência de complicações pulmonares pós-operatórias nos grupos foi aceita baseada na literatura ${ }^{10}$, cuja porcentagem é igual a $5 \%$.

Na comparação das variáveis antropométricas, tempo de circulação extracorpórea, tempo de ventilação mecânica, permanência na unidade de terapia intensiva e permanência hospitalar, utilizou-se o teste t de Student, para dados com distribuição normal ou seu equivalente Mann-Whitney.

Para comparação entre e intra grupos ao longo do tempo, utilizou-se análise de variância para medidas repetidas para dois fatores (ANOVA TWO WAY RM) e análise da variância para medidas repetidas para um fator (ANOVA ONE WAY RM). Para comparações múltiplas utilizou-se o teste Tukey.

O Teste qui quadrado (x2) foi utilizado para investigar a prevalência de complicações respiratórias.

Para todos os testes foi adotado nível de significância estatística de $5 \%$. 


\section{Resultados}

Foram recrutados 504 indivíduos para o protocolo, conforme ilustrado na Figura 1. Desses, 276 (55\%) foram excluídos por: a) presença de secreção brônquica (24\%); b) tempo de CEC maior que 120 minutos (20\%); c) relação PaO2/FiO2 menor que 200 (38\%); d) outras causas: tempo de ventilação mecânica maior que 12 horas (4\%), instabilidade hemodinâmica com necessidade de reabordagem cirúrgica (4\%), uso de assistência circulatória mecânica (6,5\%), extubação acidental (1,8\%), convulsão (0,36\%) e broncoaspiração (0,36\%). Foram incluídos 228 sujeitos, randomizados em nos grupos ASP $(n=114)$ e NASP $(n=114)$.

Características gerais dos participantes estão apresentados na Tabela 1. Houve predomínio do gênero masculino (69\% ASP e 59\% NASP). Dados antropométricos e tempos hospitalares não apresentam diferenças estatísticas significantes entre os grupos, exceto tempo de circulação extracorpórea, menor no grupo ASP $(p=0,02)$.

Figura 1. Fluxograma de estudo

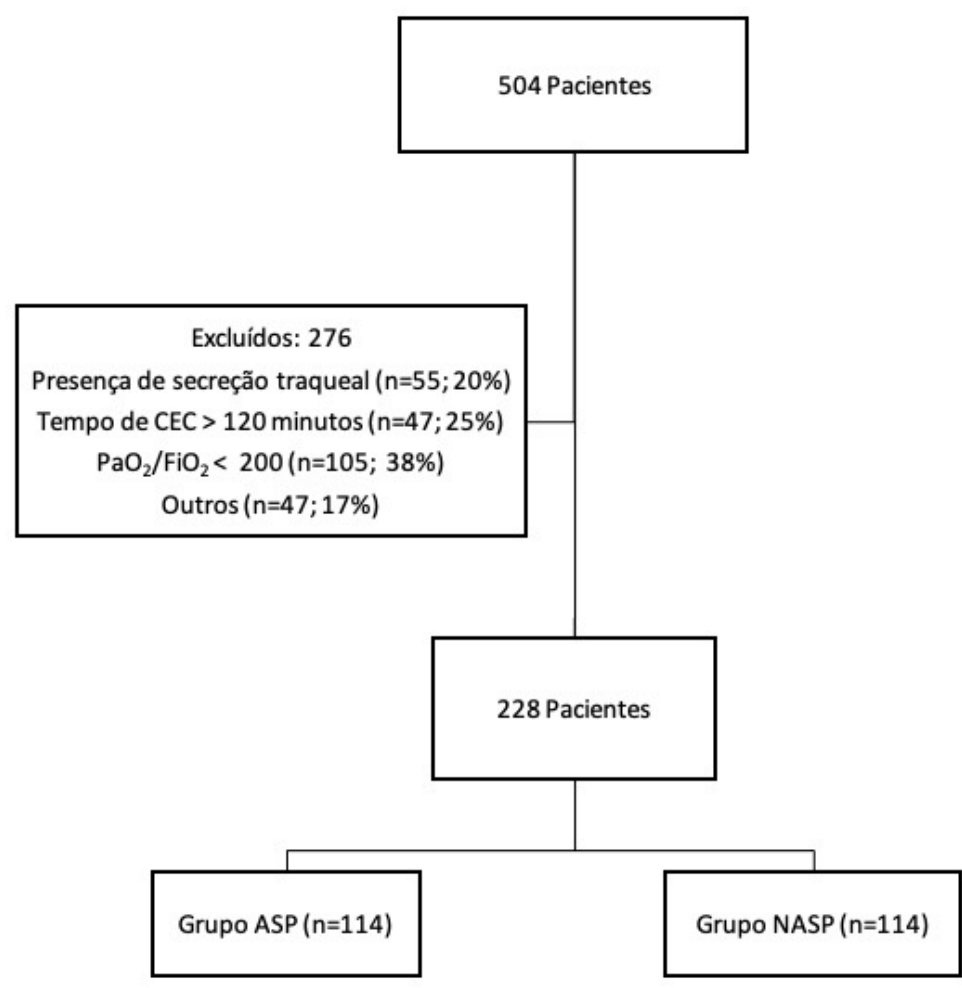

Tabela 1. Características gerais dos grupos ASP e NASP

\begin{tabular}{|c|c|c|c|}
\hline & $\operatorname{ASP}(n=114)$ & $\operatorname{NASP}(n=114)$ & $p$ \\
\hline Idade, ano & $62(55-69)$ & $60(53-65)$ & .12 \\
\hline Peso, kg & $69.35 \pm 11.65$ & $67.69 \pm 11.70$ & .30 \\
\hline Altura, cm & $1.66(1.60-1.72)$ & $1.65(1.58-1.71)$ & .16 \\
\hline Índice de Massa Corpórea, kg/m² & $25(22-27)$ & $25(22-27)$ & .93 \\
\hline Tempo de Cirurgia, minutos & $315 \pm 77$ & $330 \pm 73$ & .12 \\
\hline Tempo de CEC, minutos & $65(52-87)$ & $78(56-97)$ & $.02^{*}$ \\
\hline Tempo de Ventilação Mecânica, minutos & $315(210-390)$ & $286(220-370)$ & .22 \\
\hline Tempo de permanência em UTI, dias & $3(2-4)$ & $3(2-4)$ & .56 \\
\hline Tempo de Internação Hospitalar, dias & $8(7-12)$ & $9(7-13)$ & .96 \\
\hline
\end{tabular}




\section{Complicações pós-operatórias}

Do total dos sujeitos estudados as complicações pulmonares pós-operatórias representaram 7,8\% da amostra estudada. No grupo ASP, 5 indivíduos apresentaram traqueobronquite (8\%) e 6 pneumonias (10\%), enquanto no grupo NASP, foram 3 (5\%) e 4 (7\%) indivíduos, respectivamente, conforme ilustrado na Figura 2.

Figura 2. Valores de complicações pulmonares nos grupos ASP e NASP. Teste qui quadrado $\left(x^{2}\right)$

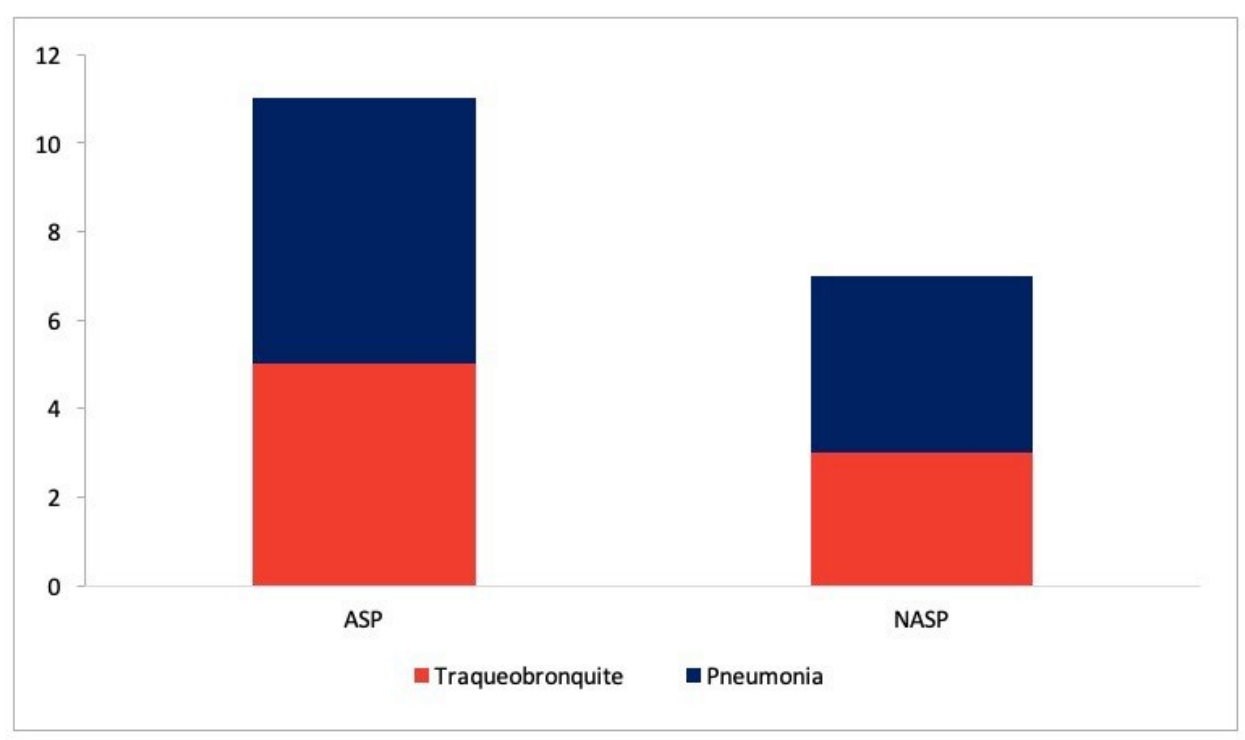

\section{Efeitos na FC e Pressão Arterial}

As variações ocorridas na frequência cardíaca, pressões arteriais sistólica, diastólica e média estão ilustradas na Figura 3.

Imediatamente após a extubação (M0), os valores de FC apresentaram elevações significantes até o $5^{\circ}$ minuto quando comparados aos valores basais, sem diferença entre os grupos $(p=0,55)$. Os valores mais altos foram registrados no $1^{\circ}$. minuto (M1), variando de 102 bpm (pré-extubação) para 111 bpm em ASP e de 105 bpm para 108 bpm em NASP.

A PAS apresentou diferença estatisticamente significante entre os grupos $(p=0,009)$. Os maiores valores foram observados em ASP nos momentos pré-extubação, imediatamente após e nos momentos seguintes à extubação até $07^{\circ}$. minuto; neste grupo os maiores valores ocorreram de $\mathrm{M} 0$ a M2, enquanto em NASP ocorreu em M3. As médias de PAD apresentaram diferenças estatisticamente significantes entre os grupos de M0 a M2. Em ASP, a PAD se elevou e em NASP houve decréscimo. A PAM apresentou significância estatística entre os grupos nos momentos M0 a M4. Em ambos os grupos seus valores voltaram aos níveis iniciais no $8^{\circ}$. minuto após a extubação (Figura 3). 


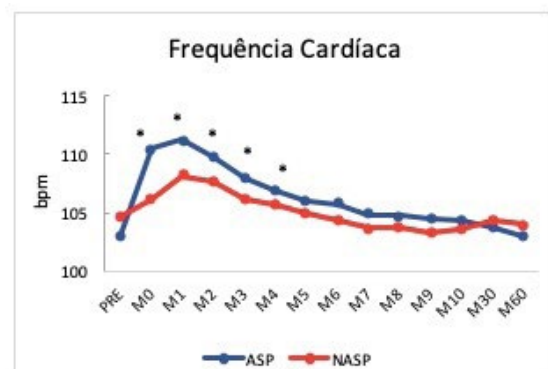

Pressão Arterial Diastólica

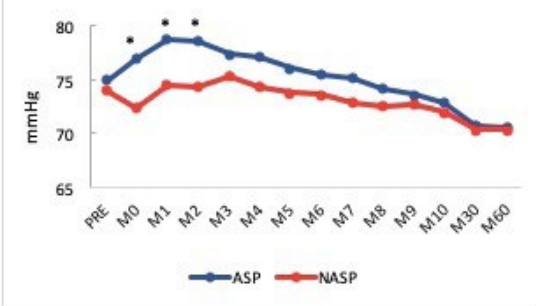

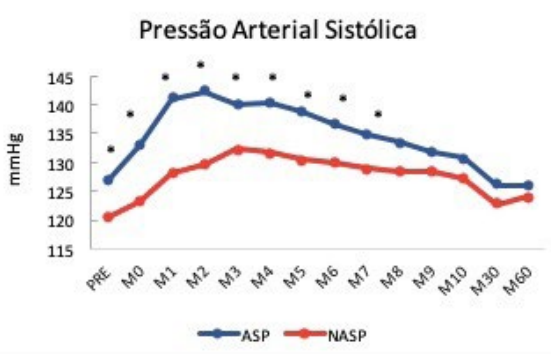

Pressão Arterial Média

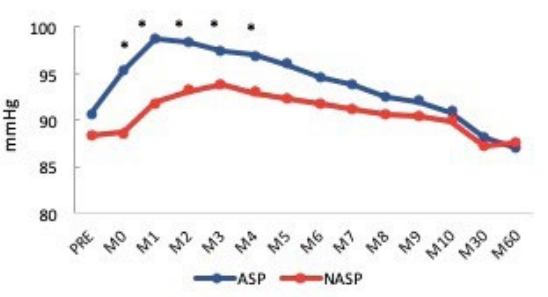

PRE: antes da extubaçăo, $M 0=$ momento da extubação, $M 1=1^{\circ}$ minuto após extubação,
$M 2=2^{\circ}$ minuto após extubação, $M 3=3^{\circ}$ minuto após extubação, $M 4=4^{\circ}$ minuto após
extubação, $M 5=5^{\circ}$ minuto após extubação, $M 6=6^{\circ}$ minuto após extubação, $M 7=7^{\circ}$
minuto após extubação, $M 8=8^{\circ}$ minuto após extubação, $M 9=9^{\circ}$ minuto após extubação,
$M 10=10^{\circ}$ minuto após extubaçăo, $M 30=30^{\circ}$ minuto após extubaçăo, $M 60=$ uma hora
após extubaçăo, ${ }^{\star} p<0,05$
Anova Two Way RM / Anova One Way RM/ Teste Tukey

\section{Efeitos na oxigenação e frequência respiratória}

As variações ocorridas na frequência respiratória e SpO2 estão ilustradas na Figura 4.

A aspiração provocou redução significante na $\mathrm{SpO} 2$ e elevação na frequência respiratória, com diferenças entre os grupos no momento pós-extubação (M0). Em ASP a SpO2 reduziu de 99,3\% para 98\% (pré x M0); nos demais momentos os valores foram significantemente menores do que na pré-extubação. Em NASP o menor valor da SpO2 foi em M1, com retorno aos valores iniciais após 60 minutos.

A frequência respiratória elevou-se em ambos os grupos imediatamente após a extubação (M0), com diferença entre os grupos ( $p=0,002$ ). Em ASP o aumento variou de 15,5 rpm para 21,6 rpm (pré x M0), mantendo-se elevada até o $4^{\circ}$. minuto; após, as reduções foram gradativas até M60 quando retornaram aos valores basais. Em NASP elevação significante ocorreu até M5, retornando aos valores basais em M10 (Figura 4). 
Figura 4. Valores ventilatórios antes e após procedimentos de extubação nos grupos ASP e NASP

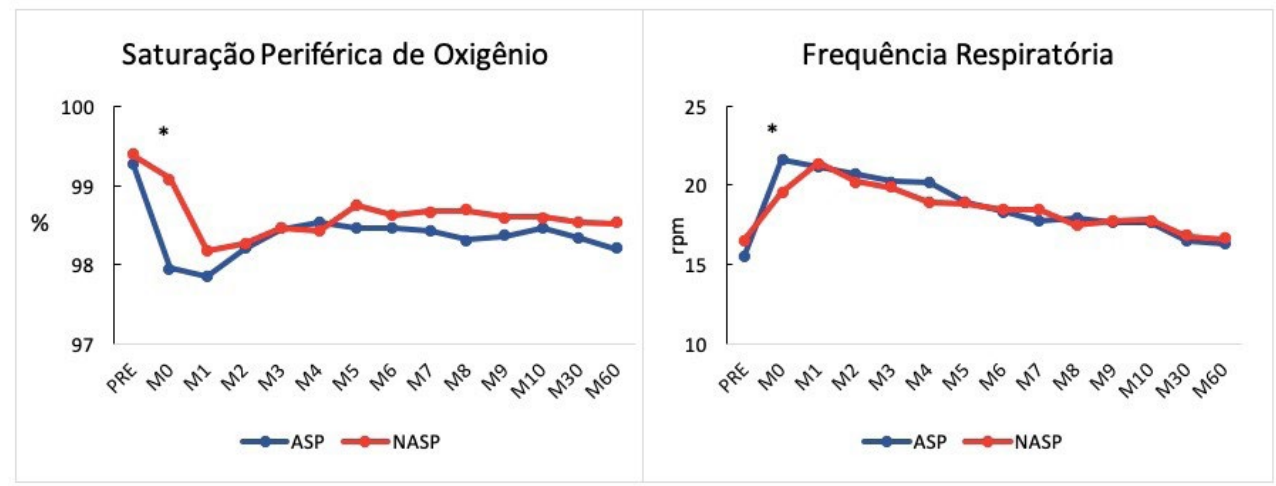

PRE: antes da extubação, $M 0=$ momento da extubação, $M 1=1^{\circ}$ minuto após extubação, $M 2=2^{\circ}$ minuto após extubação, $M 3=3^{\circ}$ minuto após extubação, $M 4=4^{\circ}$ minuto após extubação, $M 5=5^{\circ}$ minuto após extubação, $M 6=6^{\circ}$ minuto após extubação, $M 7=7^{\circ}$ minuto após extubação, $M 8=8^{\circ}$ minuto após extubação, $M 9=9^{\circ}$ minuto após extubação, $M 10=10^{\circ}$ minuto após extubação, $M 30=30^{\circ}$ minuto após extubação, $M 60=$ uma hora após extubação, * $p<0,05$

Anova Two Way RM / Anova One Way RM/ Teste Tukey

\section{Custos e Tempo de Procedimento}

Na época do estudo, o procedimento de aspiração teve um custo de material calculado de $\mathrm{R} \$ 7,23$ para cada paciente do grupo ASP, sendo: 1 par de luva estéril: $R \$ 1,45,2$ pares de luva de procedimento: $R \$$ $0,40,2$ máscaras cirúrgicas: $R \$ 0,12,1$ seringa de $20 \mathrm{ml}$ : $\mathrm{R} \$ 0,27,1$ sonda de aspiração com válvula número 12: $\mathrm{R} \$ 0,39$ e 1 coletor de secreção $500 \mathrm{ml}: \mathrm{R} \$ 4,60$. Gerando um custo total de $\mathrm{R} \$ 824,22$. No grupo NASP o custo individual foi de $R \$ 0,79$, sendo 2 pares de luva de procedimento: $R \$ 0,40,2$ máscaras cirúrgicas: $R \$$ 0,12 e 1 seringa: $R \$ 0,27$ totalizando $R \$ 90,06^{10}$.

O tempo despendido nos sujeitos do grupo ASP foi de 2 minutos e 68 segundos por paciente, $357 \mathrm{mi}-$ nutos e 20 segundos por profissional, totalizando 714 minutos e 40 segundos ou 12 horas 30 minutos e 6 segundos, tendo em vista que o tempo despendido por profissional foi multiplicado por 2 pois o procedimento de aspiração foi realizado por 2 profissionais. No grupo NASP o procedimento despendeu 28 segundos por paciente, 53 minutos e 20 segundos por profissional, não havendo necessidade de dois profissionais.

\section{Discussão}

Em nosso estudo, indivíduos sem sinais de secreção brônquica, foram extubados sem receberem intervenção de aspiração traqueal. Esta conduta não influenciou a incidência de complicações pulmonares e o tempo de permanência hospitalar. Por outro lado, aspiração traqueal acarretou maior estresse cardiovascular, envolveu maior tempo profissional e gerou custos mais elevados.

Disfunções pulmonares são frequentes no pós-operatório imediato de cirurgia cardíaca. As mais comuns são as atelectasias e os derrames pleurais, decorrentes, principalmente, da esternotomia, uso de drogas anestésicas, tempo de ventilação mecânica e abertura da cavidade pleural.

A incidência de complicação pulmonar, definida como aquela que produz doença identificável ou disfunção significante que afeta a evolução clínica, tende a ser menor no pós-operatório de cirurgia cardíaca. $\mathrm{Na}$ instituição que foi realizado o estudo essas complicações são classificadas em traqueobronquite e pneumonia, por critérios estabelecidos pela Comissão de Infecção Hospitalar? 
Dados institucionais mostram a incidência de infecção pulmonar pós-operatória está entre 5,3 a 9,911. Outros estudos, com sujeitos recebendo assistência fisioterapêutica, mostram incidência de infecção pulmonar entre $7,1 \%^{12}$ e $10 \%^{13}$ no pós- operatório de revascularização do miocárdio. Em cirurgia valvar, $11 \%$ dos sujeitos considerados de baixo risco cirúrgico, desenvolveram infecção pulmonar ${ }^{14}$.

Nossa amostra representa esse universo de indivíduos operados anualmente. Independente do uso da aspiração previamente à extubação, a taxa de infecção não se alterou. Assistência fisioterapêutica é aplicada, de rotina, em todos os sujeitos cirúrgicos para melhorar volumes pulmonares e manter vias aéreas livres de secreção brônquica, o que pode influenciar nos valores de infecção pulmonar. Sabe-se que, indivíduos submetidos à cirurgia cardíaca apresentam, em sua maioria, distúrbio ventilatório restritivo, com perda de volumes e capacidades pulmonares, é possível que a presença de infecção pulmonar esteja associada a outros fatores, incluindo tabagismo, alcoolismo e o grau de insuficiência cardíaca prévio ao ato cirúrgico ${ }^{15}$.

A ausência de secreção brônquica detectável justificou a conduta de extubar os indivíduos sem utilizar a aspiração endotraqueal. A evolução clínica desses indivíduos confirmou que o procedimento não tinha indicação e, portanto, foi desnecessário.

Os tempos hospitalares foram semelhantes entre os grupos, variando de 9 a 17 dias de internação pósoperatória, também em consonância com os dados institucionais ${ }^{11}$. Causas hemodinâmicas e pulmonares retardam a alta hospitalar. Os sujeitos do estudo apresentavam critérios pré-operatórios que os classificavam como de baixo risco cirúrgico com provável evolução clinica livre de intercorrências. Embora $24,5 \%$ deles desenvolvessem algum tipo de complicação pós-operatória, a magnitude desta disfunção não foi suficientemente grande para alterar sua evolução e seu tempo de estadia hospitalar.

\section{Efeitos agudos da aspiração}

A extubação é um procedimento que altera o sistema cardiorrespiratório. Observamos que, independente do grupo, a extubação provocou aumentos na frequência cardíaca e pressão arterial, principalmente nos 5 primeiros minutos. No entanto, os maiores aumentos ocorreram durante a aspiração traqueal.
Este fato ocorre, em parte, pela inserção da sonda de aspiração na região traqueal, local de grande número de mecanorreceptores ${ }^{16}$. A irritação, provocada nas vias aéreas, pode desencadear oscilação hemodinâmica pela modulação autonômica, com respostas de aumento da FC. Outros efeitos como estimulação vagal, broncoespasmo, redução da oferta de oxigênio aos pulmões e microatelectasias tem sido descritos ${ }^{17}$. Tais repercussões não foram encontradas em nosso estudo. Porém, a possibilidade dessas ocorrências torna o procedimento seletivo e recomendado somente quando necessário.

Na população do estudo também não observamos ocorrência de efeitos adversos, incluindo arritmias cardíacas. A suplementação de oxigênio $(\mathrm{FiO} 2=1)$, durante a aspiração, pode ser um fator que contribui para evitar hipoxemia e, consequentes arritmias ${ }^{18}$. As variações observadas na oxigenação de nossos indivíduos foram pequenas, não sendo registrados níveis hipoxêmicos. Por outro lado, a hiperoxigenação, embora previna a hipoxemia, não impede o efeito da pressão negativa do vácuo e da desconexão do aparelho de ventilação mecânica sobre o volume pulmonar causando microatelectasias.

No pós-operatório de cirurgia cardíaca, a monitorização da pressão arterial deve ser contínua por ser um dos sinais vitais que indicam precocemente alterações da função cardiovascular ${ }^{18}$. $\mathrm{O}$ ato de tossir, comum durante os procedimentos de aspiração e extubação, pode causar taquicardia, hipertensão e liberação de catecolaminas em excesso, aumentando assim a demanda de oxigênio ao miocárdio ${ }^{19,20}$.

Nossos resultados mostram que, no momento da extubação, a PAD aumentou no grupo ASP e reduziu no grupo NASP, porém imediatamente após, houve aumentos dos valores em ambos os grupos. A pressão positiva, utilizada na ventilação mecânica, aumenta a pressão pleural e, consequentemente, a pressão da região pericárdica. Pressões geradas dentro do saco pericárdico podem ser responsáveis por restrição diastólica do ventrículo esquerdo, diminuindo globalmente as pressões transmurais das câmaras cardíacas com repercussão na função ventricular diastólica. A retirada da pressão positiva provoca retorno da pressão pleural para valores subatmosféricos, com descompressão das veias cavas e aumento do retorno venoso, resultando em maior enchimento das câmaras cardíacas e elevação do débito cardíaco ${ }^{21}$. 
Com a diminuição da pressão intratorácica e aumento do débito cardíaco, as pressões arteriais sofrem elevações imediatas, conforme mostram nossos resultados. No entanto, ao longo do tempo, as pressões tendem a se normalizar, pela maior sensação de conforto referida pelo paciente, maior controle da dor e efeito de drogas analgésicas 22 .

Observamos que no grupo ASP a frequência respiratória se elevou de modo significativo. É possível que com a aspiração, a pressão negativa do vácuo gere colapsos pulmonares de diferentes magnitudes e a frequência respiratória se eleve para manter a ventilação constante. No entanto, em ambos os grupos, houve aumento da frequência respiratória, o que pode ser causado, em parte, pela perda de volume pulmonar após a retirada do suporte pressórico, dor pós-operatória, alteração mecânica restritiva incisional, presença de drenos, dentre outros 23,24 .

A avaliação criteriosa da real necessidade da técnica de aspiração traqueal em sujeitos no pós-operatório imediato de cirurgia cardíaca evita a prática rotineira, possibilita redução do custo e otimização do tempo na jornada de trabalho do fisioterapeuta, tendo em vista quando se opta por este procedimento o tempo despendido e os custos dos materiais são bem mais elevados.

A aspiração endotraqueal implica em riscos imediatos ao paciente. Fazer bem (beneficência) e sem riscos (não maleficência) são aspectos éticos envolvidos no cuidado em saúde. O procedimento com menor risco/benefício deve ser escolhido na prática assistencial para que riscos desnecessários e escolhas não éticas sejam evitados.

Por esses dados, a aspiração traqueal de rotina deve ser questionada e estudada, para que sua indicação seja seletiva e baseada na real necessidade. Caso contrário, passa a ser um procedimento invasivo, que gera riscos ao paciente e aumento custo na saúde.

As limitações do estudo foram: a) ausência de disponibilidade de monitor de débito cardíaco para analisar variáveis hemodinâmicas (FC, PAS, PAD e PAM) e débito cardíaco. $O$ equipamento era destinado apenas aos mais graves, que não atendiam aos critérios de inclusão do estudo; b) ausência de gasometria antes da saída do paciente do centro cirúrgico. Gasometria base para avaliação da inclusão no estudo coletada na admissão do paciente na UTI, não levando em consideração as possíveis alterações ventilatórias do transporte do centro cirúrgico para UTI; c) complicações pulmonares baseadas em registros médicos em prontuário eletrônico.

\section{Conclusão}

Em nosso estudo, a supressão da aspiração traqueal previamente à extubação, em indivíduos no pós-operatório imediato de cirurgia cardíaca e sem sinais de secreção brônquica, não interferiu na incidência de complicações pulmonares infecciosas e no período de internamento hospitalar. Por outro lado, otimizou tempo profissional e reduziu custos.

Secundariamente, a extubação per si provocou alterações hemodinâmicas e respiratórias, sem repercussões clínicas. A adição da aspiração endotraqueal potencializou essas alterações e gerou maior custo.

\section{Contribuições das autoras}

Campos A participou da concepção, delineamento, coleta de dados da pesquisa e redação do artigo. Rabelo $A B L$ participou da busca e coleta de dados, revisão de literatura e redação do artigo. Barros JO participou da busca e análise estatística dos dados, interpretação de resultados, revisão de literatura, coleta de dados e redação. Nozawa E participou do delineamento do estudo e revisão crítica do manuscrito. Hajjar LA e Galas FRG participaram da revisão crítica do manuscrito. Feltrim MIZ participou da concepção, delineamento, análise estatística e interpretação dos dados e redação do artigo.

\section{Conflitos de interesses}

Nenhum conflito financeiro, legal ou político envolvendo terceiros (governo, empresas e fundações privadas, etc.) foi declarado para nenhum aspecto do trabalho submetido (incluindo, mas não se limitando a subvenções e financiamentos, participação em conselho consultivo, desenho de estudo, preparação de manuscrito, análise estatística, etc.). 


\section{Referências}

1. Day T, Farnell S, Haynes S, Wainwright $S$, Wilson-Barnett J. Tracheal suctioning: an exploration of nurses' knowledge and competence in acute and high dependency ward areas. J Adv Nurs. 2002;39(1):35-45. doi: 10.1046/j.1365-2648.2002.02240.x

2. Day T, Farnell S, Wilson-Barnett J. Suctioning: a review of current research recommendations. Intensive Crit Care Nurs. 2002;18(2):79-89. doi: 10.1016/s0964-3397(02)00004-6

3. Jelic S, Cunningham JA, Factor P. Clinical review: Airway hygiene in the intensive care unit. Crit Care. 2008;12(2):209. doi: 10.1186/ cc6830

4. Lucchini A, Zanella A, Bellani G, Gariboldi R, Foti G, Pesenti A et al. Tracheal Secretion Management in the Mechanically Ventilated Patient: Comparison of Standard Assessment and an Acoustic Secretion Detector. Respir Care. 2011;56(5):596-603. doi: 10.4187/ respcare.00909

5. Restrepo RD, Brown JM, Hugles JM. American Association for Respiratory Care. AARC Clinical Practice Guidelines. Endotracheal suctioning of mechanically ventilated patients with artificial airways. Respir Care 2010;55(6):758-64.

6. Favretto DO, Silveira RCCP, Canini SRMS, Garbin LM, Martins FTM, Dalri MCB. Endotracheal suction in intubated critically ill adult patients undergoing mechanical ventilation: a systematic review. Rev Latino-Am Enfermagem. 2012;20(5):997-1007. doi: $\underline{10.1590 / S 0104-11692012000500023}$

7. Celik SS, Elbas NO. The standard of suction for patients undergoing endotracheal intubation. Intensive Crit Care Nurs. 2000;16(3):191-8. doi: 10.1054/iccn.2000.1487

8. Vanner R, Bick E. Tracheal pressures during open suctioning. Anaesthesia. 2008;63(3):313-5. doi: 10.1111/j.13652044.2007.05348.x

9. Subcommittee of hospital infection control of Heart Institute Medical School of the University of Sao Paulo [Internet]. 2014 [cited 7 November 2014]. Available from: www.incor.usp.br/sites/ incor2013/index.php/component/content/article/197-intranet/ manuais-normas-e-rotinas/comissoes/697-intranet-manuaisnormas-e-rotinas-comissoes-subcomissao-de-controle-de-infeccao

10. Department of Material Division of Heart Institute Medical School of the University of Sao Paulo. [Internet]. 2014. [accessed in 2014 june 04]. Available from: http://www.hc.fm.usp.br/images/ pdf/nilo/compras/relacaocompras_hcfmusp_2014/Relacao_de_ compras」AN-2014.pdf

11. Institut de Cardiologie de Montréal. Annual Activities Report oh Heart Institute 2014 [Internet]. 2014 [accessed in 2014 dec 21]. Available from: https://www.icm-mhi.org/sites/default/files/docs/ ficm/corrige_icm_annual_report_2014-15.pdf

12. Stiller K, Jenkins S, Hall B. Efficacy of Breathing and Coughing Exercises in the Prevention of Pulmonary Complications After
Coronary Artery Surgery: To the Editor. Chest. 1995;107(2):587588. doi: 10.1378/chest.107.2.587-a

13. Jenkins SC, Soutar SA, Loukota JM, Johnson LC, Moxham J. Physiotherapy after coronary artery surgery: are breathing exercises necessary? Thorax. 1989;44(8):634-639. doi: 10.1136/thx.44.8.634

14. Franco SS, Malbouisson LMS, Grinberg M, Feltrim MIZ. A propose of pulmonary dysfunction stratification afther valve surgery by physiotherapeutic assistance level. Revista Brasileira de Cirurgia Cardiovascular. 2015;30(2):188-97. doi: 10.5935/16789741.20150006

15. Lawrence VA, Cornell JE, Smetana GW. Strategies To Reduce Postoperative Pulmonary Complications after Noncardiothoracic Surgery: Systematic Review for the American College of Physicians. Ann Intern Med. 2006;144(8):596-608. doi: 10.7326/0003-4819-144-8-200604180-00011

16. Brucia J, Rudy E. The effect of suction catheter insertion and tracheal stimulation in adults with severe brain injury. Heart \& Lung. 1996;25(4):295-303.

17. Kerem E, Yatsiv I, Goitein KJ. Effect of endotracheal suctioning on arterial blood gases in children. Intensive Care Med. 1990;16(2):95-99.

18. Dias FS, Rezende E, Mendes CL, Réa-Neto A, David CM, Schettino $\mathrm{G}$ et al. Part II: basic hemodynamic monitoring and the use of pulmonary artery catheter. Rev Bras Ter Intensiva. 2006;18(1):63-77. doi: 10.1590/S0103-507X2006000100012

19. Paulissian R, Salem MR, Joseph NJ, Braverman B, Cohen HC, Crystal GJ et al. Hemodynamic Responses to Endotracheal Extubation After Coronary Artery Bypass Grafting. Anesth Analg. 1991;73(1):10-5. doi: 10.1213/00000539-199107000-00003

20. Carlos RV, Bittar CS, Lopes MR, Auler Júnior JOC. Variation of systolic blood pressure as a method of diagnosis of hypovolemia during anesthesia for cardiac surgery. Rev Bras Anestesiol. 2005;55(1):3-18. doi: 10.1590/S0034-70942005000100002

21. André AC, DelRossi A. Hemodynamic management of patients in the first 24 hours after cardiac surgery. Crit Care Med. 2005;33(9):2082-93. doi: 10.1097/01.ccm.0000178355.96817.81

22. Giacomazzi CM, Lagni VB, Monteiro MB. Postoperative pain as contributor to pulmonary function inpairment in patients submitted to heart surgery. Braz J Cardiovasc Surg. 2006;21(4):386-92. doi: 10.1590/S0102-76382006000400008

23. Guizilini S, Gomes WJ, Faresin SM, Bolzan DW, Buffolo $\mathrm{E}$, Carvalho AC et al. Influence of Pleurotomy on Pulmonary Function After Off-Pump Coronary Artery Bypass Grafting. The Annals of Thoracic Surgery. 2007;84(3):817-822. doi: 10.1016/j. athoracsur.2007.04.062

24. Maggiore SM, Lellouche F, Pignataro C, Girou E, Maitre B, Richard JCM et al. Decreasing the Adverse Effects of Endotracheal Suctioning During Mechanical Ventilation by Changing Practice. Respir Care. 2013;58(10):1588-97. doi: 10.4187/respcare.0226 